\title{
Surgical Management of Corneal Hydrops: Case Series
}

\author{
๑ Gökçen Özcan, ๑ Ömür Özlenen Uçakhan \\ Ankara University Faculty of Medicine, Department of Ophthalmology, Ankara, Turkey
}

\begin{abstract}
Descemet's membrane (DM) rupture/detachments have traditionally been treated conservatively, with limited efficacy and a long rehabilitation period that significantly affects patients' vision and quality of life. Although there are no established gold standards for the timing and nature of treatment, with this series of 4 cases we aimed to highlight the importance of the current optimal intervention methods. The first two patients were treated with anterior chamber injection of isoexpansile $14 \% \mathrm{C} 3 \mathrm{~F} 8$ due to acute hydrops associated with keratoglobus in the first case and keratoconus in the second case. The third patient had keratoglobus and chronic hydrops complicated by multiple stromal clefts detected on anterior segment optical coherence tomography, and the fourth patient had a chronic broad DM detachment which occurred after cataract surgery. Both of these patients were treated with intracameral C3F8 injection together with corneal compressive sutures. In all four cases, DM reattached completely and effectively with surgical intervention. Surgical management of DM rupture/detachment with intracameral gas injection and compressive corneal sutures seems to provide fast symptomatic relief and less healing-related corneal scarring with better visual rehabilitation, and may alleviate the need for corneal transplant surgery in this group of patients.

Keywords: Descemet's membrane detachment, Descemet's membrane rupture, acute hydrops, Descemetopexy, 14\% isoexpansile C3F8, corneal compression sutures
\end{abstract}

\section{Introduction}

Descemet's membrane (DM) integrity problems such as ruptures or detachments manifest with loss of vision due to corneal edema and DM folds. Rupture of the DM has been reported in association with keratoglobus, keratoconus, congenital glaucoma, intraocular surgeries, prolonged and complicated labor with forceps, Terrien marginal degeneration, Acanthamoeba keratitis, and rarely spontaneously. ${ }^{1,2,3,4,5}$

Acute corneal hydrops is characterized by a rupture in the DM in the setting of corneal ectasia, which results from stretching of the DM leading to its rupture, allowing aqueous to enter the corneal stroma and epithelium. Corneal hydrops is relatively uncommon and is estimated to occur in $2.6-2.8 \%$ of patients with keratoconus. With a male preponderance, the mean age at onset of corneal hydrops is around 25 years. ${ }^{6}$ Although the development of acute hydrops in keratoconus is well described, acute hydrops secondary to keratoglobus is rare in the literature.

On the other hand, Descemet's membrane detachment (DMD) mainly occurs in association with intraocular surgeries, particularly cataract surgery. Possible mechanisms by which DMD develops during intraocular surgeries have been reported as shallow anterior chamber; complicated or repeated surgeries; inadvertent insertion of instruments or inadvertent injection of saline and viscoelastic between the corneal stroma and DM; anteriorly located and shelved incisions; and the use of dull blades. ${ }^{7,8}$

\footnotetext{
Address for Correspondence: Gökçen Özcan, Ankara University Faculty of Medicine, Department of Ophthalmology, Ankara, Turkey E-mail: drgokcencondu@gmail.com ORCID-ID: orcid.org/0000-0002-2616-5941

Received: 25.02.2021 Accepted: 01.10.2021
}

Cite this article as: Özcan G, Özlenen Uçakhan Ö. Surgical Management of Corneal Hydrops: Case Series. Turk J Ophthalmol 2022;52:64-68

${ }^{\circ}$ Copyright 2022 by Turkish Ophthalmological Association Turkish Journal of Ophthalmology, published by Galenos Publishing House. 
Complete spontaneous resolution of edema associated with DM separation may take months, with variations from 5 to 36 weeks. $^{6}$ In some cases, prolonged edema may lead to inflammatory reaction and neovascularization, affecting the prognosis of subsequent transplantation procedures. ${ }^{9}$ Although DM integrity problems spontaneously resolve, severe visual symptoms or vision loss and long disease duration negatively influence quality of life and cause significant visual morbidity.

Conventional treatments for DM integrity problems, such as patching or bandage contact lens, tarsorrhaphy, cycloplegia, or hypertonic ophthalmic solutions, are of limited efficacy. Shaw ${ }^{10}$ attempted corneal cauterization and thermokeratoplasty for faster resolution of corneal edema. Hirst and Dejuan ${ }^{11}$ presented a technique using tissue adhesives and viscoelastic agents. Macsai and Lemley ${ }^{12}$ described onlay epikeratoplasty with a donor corneoscleral button. More recently, Hussin et al. ${ }^{13}$ described a novel technique, non-expansile $(14 \%)$ perfluoropropane $\left(\mathrm{C}_{3} \mathrm{~F}_{8}\right)$ gas tamponade "descemetopexy," which currently seems to be the most widely used technique. Rajaraman and associates ${ }^{14}$ combined the use of intracameral gas and compression sutures in corneal hydrops with stromal clefts and reported it to be an effective and safe treatment modality in the presence of large gaping tears and stromal clefts. Mohebbi et al. ${ }^{15}$ indicated that combined intracameral gas injection and approximation sutures provided rapid recovery with very rare complications for the treatment of acute corneal hydrops. Zhao et al. ${ }^{16}$ reported superior clinical outcomes using compression sutures with intracameral air injection versus thermokeratoplasty in the management of acute corneal hydrops. Our purpose in this report was to present a case series of 4 eyes with DM integrity problems and describe our approach to management.

\section{Case Reports}

\section{Case 1}

A 47-year-old woman presented with 6-day history of blurred vision and pain in the left eye. Corrected distance visual acuities (CDVA) were 20/200 in the right eye and counting fingers at $50 \mathrm{~cm}$ in the left eye. Anterior segment examination revealed keratoglobus bilaterally and acute hydrops with central corneal edema in the left eye (Figure 1a). Pentacam HR (Oculus Optikgerate $\mathrm{GmbH}$, Wetzlar, Germany) anterior segment tomography revealed keratoglobus with a maximum keratometry measurement (KMax) of 75.7 diopters (D) and thinnest corneal thickness (TCT) of $160 \mu \mathrm{m}$ in the right eye. Anterior-segment optical coherence tomography (AS-OCT, Visante, Carl Zeiss Meditec, Dublin, CA) revealed a giant DMD centrally (Figure 1b). Intracameral injection of an isoexpansile mixture of $14 \%$ $\mathrm{C}_{3} \mathrm{~F}_{8}$ (gas diluted with air) was performed in the operating room after anterior chamber paracentesis. Three injections were done in a period of 2 months to completely reattach the DMD, and complete resolution of edema took 3 months. The decision to reinject was made when corneal edema increased and DMD enlarged on AS-OCT.
At 1-year follow-up examination, CDVA was 20/200 in the left eye. A slight stromal scar was observed, and the DM remained attached (Figure 1c). TCT was $138 \mu \mathrm{m}$. After genetic consultation, a diagnosis of brittle cornea syndrome was made with detection of a ZNF469 mutation. Other systemic findings of the patient were sensorineural hearing loss, metacarpal hypoplasia, and subluxation of various joints (Figure 1d).

\section{Case 2}

A 60-year-old man with keratoconus and no history of ocular surgery presented with blurred vision and pain for 7 days. CDVA was counting fingers at $50 \mathrm{~cm}$ in the right eye and 20/100 in the left eye. On slit-lamp examination, acute hydrops with bullous edema was observed in the right eye (Figure 2a). Pentacam HR showed a KMax value of $48.7 \mathrm{D}$ and TCT of $465 \mu \mathrm{m}$ in the left eye. On AS-OCT, a break in the DM with DMD was visible below the area of edema (Figure 2b). A single intracameral injection of an isoexpansile mixture of $14 \% \mathrm{C}_{3} \mathrm{~F}_{8}$ was performed and the edema had resolved at 2-week follow-up. At 3-month follow-up examination, CDVA was 20/125 and a faint central corneal scar with complete resolution of stromal edema was observed on slit-lamp examination (Figure 2c,d). Pentacam HR revealed a KMax value of $64.8 \mathrm{D}$ and a TCT of $396 \mu \mathrm{m}$ in the right eye.

\section{Case 3}

A 44-year-old man presented with complaints of blurred vision, photophobia, and pain in the right eye for 3 months. CDVA was counting fingers at $15 \mathrm{~cm}$ in the right eye and 20/40 in the left eye. Anterior segment examination showed acute hydrops with prevalent corneal edema in the right eye and a very thin ectatic cornea in the left eye (Figure 3a). Pentacam HR indicated keratoglobus with a KMax value of 61.9 D and TCT of $500 \mu \mathrm{m}$ in the right eye. AS-OCT revealed a wide



Figure 1. Case 1: Slit-lamp photos of a patient with brittle cornea syndrome showing acute hydrops with central corneal edema in the left eye (a). On anterior segment optical coherence tomography (AS-OCT), central corneal edema and Descemet's membrane detachment was observed (*) (b). Three months later, clearing of the corneal cloudiness with only slight stromal scarring was observed on slit-lamp examination (c). Hypoplasia of the fourth and fifth metacarpals was apparent in both hands $(\mathrm{d})$ 


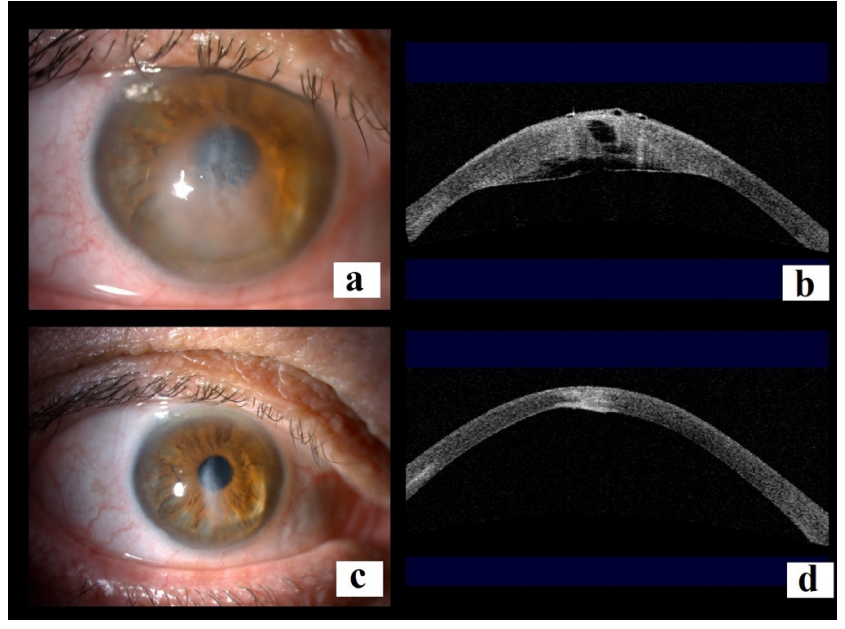

Figure 2. Case 2: Slit-lamp photos of a keratoconus patient showed acute hydrops and microcystic edema in the right eye (a). On anterior segment optical coherence tomography (AS-OCT), Descemet's membrane (DM) rupture was visible below the area of edema (b). At 3 months after $\mathrm{C}_{3} \mathrm{~F}_{8}$ administration, central corneal scar with complete resolution of stromal edema was observed

central separation of the DM from the corneal stroma, most severe temporally, with multiple stromal clefts (Figure 3b). An intracameral injection of $0.2 \mathrm{~mL}$ isoexpansile $(14 \%) \mathrm{C}_{3} \mathrm{~F}_{8}$ and seven compression sutures were applied simultaneously for the large gaping DM tear. On day 10 after the intervention, the edema was decreased and the cornea regained much of its clarity (Figure 3c). AS-OCT demonstrated complete reattachment of the DM (Figure 3d). Resolution of edema took 4 weeks. At 1-year follow-up examination, CDVA was 20/70 in the right eye. On slit-lamp examination, neither corneal edema nor neovascularization was observed, and AS-OCT showed the DM remained attached and the clefts had collapsed entirely (Figure $3 \mathrm{e}, \mathrm{f})$.

\section{Case 4}

An 88-year-old man who underwent cataract surgery in the left eye 1 year earlier at another clinic and had progressive visual deterioration since then was referred to our clinic for a corneal transplant. At initial examination, CDVA was 20/40 in the right eye and counting fingers at $10 \mathrm{~cm}$ in the left eye. Anterior segment examination revealed a very cloudy and thick cornea, obscuring the anterior chamber view. AS-OCT revealed a wide separation of the DM from the stroma (Figure 4a). An intracameral injection of $0.2 \mathrm{~mL}$ isoexpansile $(14 \%) \mathrm{C}_{3} \mathrm{~F}_{8}$ was performed together with 11 compression sutures simultaneously and resolution of edema took 4 weeks. At the 3-month followup examination, CDVA was 20/200 in the left eye, the bullous edema had resolved completely, and the DM was attached as confirmed with AS-OCT (Figure 4b,c).

\section{Discussion}

We presented four cases of DM rupture/detachment and their management. As causative factors, two of the four patients

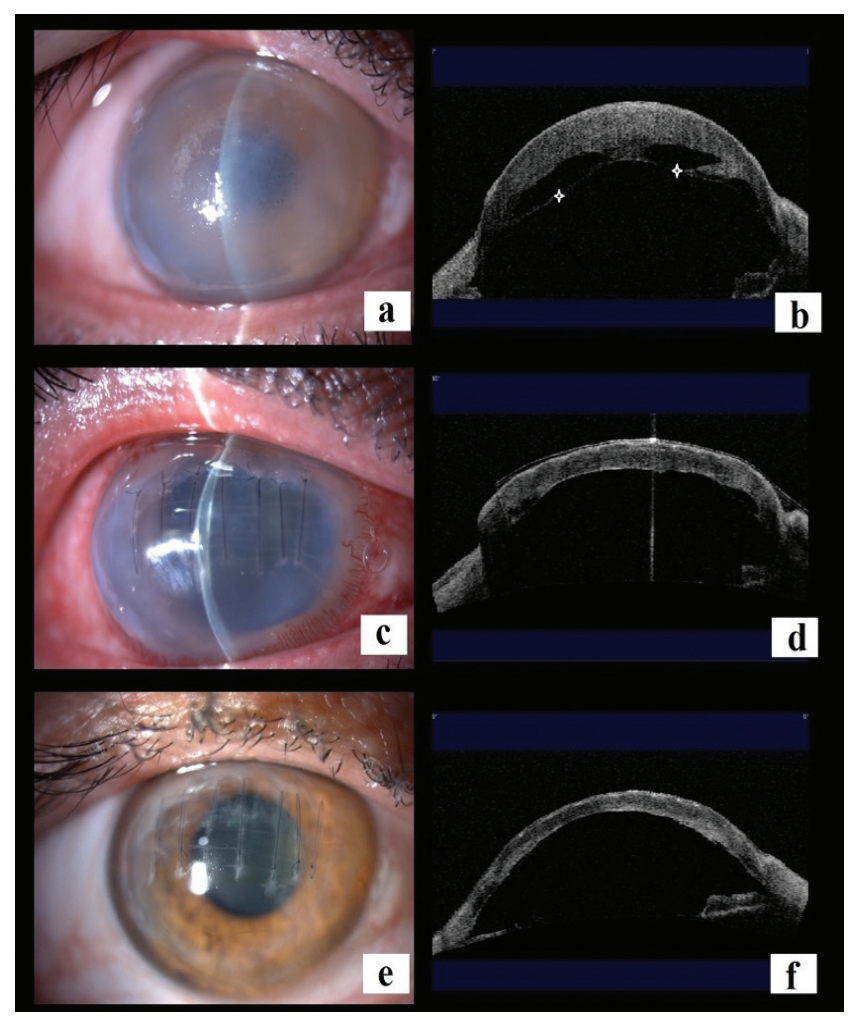

Figure 3. Case 3: Slit-lamp photos of the patient with acute hydrops and severe epithelial and stromal edema in the right eye (a). Anterior segment optical coherence tomography (AS-OCT) revealed widespread corneal edema and Descemet's membrane detachment $(\mathrm{DM})(*)$ and $\mathrm{DM}$ rupture complicated with stromal clefts (b). At 10 days after C3F8 infection together with full-thickness corneal compression sutures, the corneal haze had subsided and AS-OCT showed complete reattachment of the DM to the stroma and reduction in corneal steepness $(\mathrm{c}, \mathrm{d})$. At 1-year follow-up, the cornea was clear and the DM remained attached (e,f)

had keratoglobus, one had keratoconus, and the last patient had undergone cataract surgery. Only intracameral $\mathrm{C}_{3} \mathrm{~F}_{8}$ injection was performed in the first two cases; the third case with chronic DM rupture complicated with intrastromal clefts and the fourth case with chronic DMD were effectively managed with intracameral $\mathrm{C}_{3} \mathrm{~F}_{8}$ injection and compression sutures.

Descemetopexy with repeated injections of intracameral air or gas can accelerate the resolution of corneal edema by acting as a mechanical barrier preventing the entry of aqueous humor into the stroma and acting as a tamponading agent. ${ }^{17}$ Miyata et al. ${ }^{18}$ reported that corneal edema persisted for an average of 20 days in patients managed with intracameral air injection versus 65 days in patients who received no treatment for acute hydrops in keratoconus. As air is absorbed in a short period of time, Panda et al. ${ }^{19}$ reported descemetopexy with injection of $0.1 \mathrm{~mL}$ of an isoexpansile concentration of $20 \% \mathrm{SF}_{6}$ as an alternative. The authors reported earlier and more effective resolution of corneal edema compared to conservative management, with complete resolution achieved at 4 weeks in the $\mathrm{SF}_{6}$-injected group compared to 12 weeks in the conservative treatment group. Whereas undiluted $\mathrm{C}_{3} \mathrm{~F}_{8}$ expands to 4 times its initial volume in 4 days inside the anterior chamber, the $14 \%$ nonexpansile 


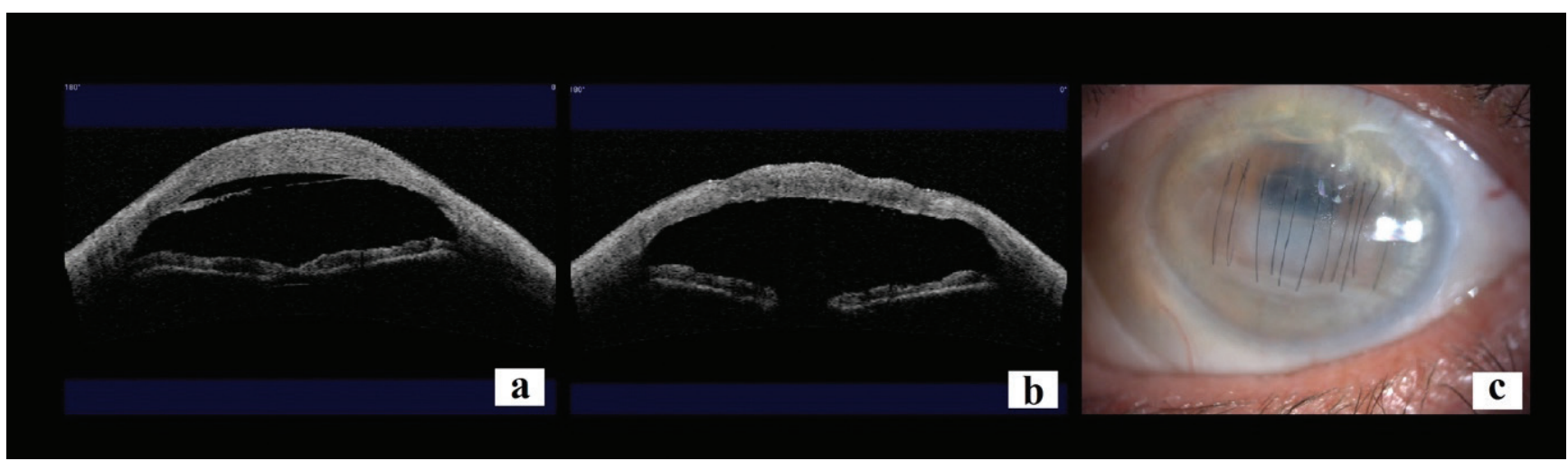

Figure 4. Case 4: Anterior segment optical coherence tomography (AS-OCT) at presentation 12 months after phacoemulsification showed corneal edema and Descemet's membrane detachment (DM) (a). At 3-month follow-up, central corneal scarring was observed without edema. On AS-OCT, the DM was totally attached and anterior corneal surface irregularities were noted $(\mathrm{b}, \mathrm{c})$

dilution of the gas persists in the anterior chamber for 6 weeks, and is therefore considered to be a safe and effective modality for early resolution of corneal edema in eyes with acute hydrops. ${ }^{20}$ In one study, descemetopexy with $1.8 \%$ sodium hyaluronate was also used to unscroll and reattach recalcitrant DMD. ${ }^{21}$

The use of intracameral $\mathrm{C}_{3} \mathrm{~F}_{8}$ with full-thickness corneal compression sutures to bring the edges of the DM tear together was also proposed. ${ }^{14}$ Suturing together with intracameral gas injection decreases the amount and number of gas fillings needed and reduces the complications associated with isoexpansile gases such as pupillary block glaucoma, endothelium toxicity, and cataract formation. ${ }^{22}$

An AS-OCT study of cases involving acute hydrops described two stages of resolution: DM reattachment and endothelial migration. When DM breaks, it retracts or coils. First, the DM has to reattach to the posterior stroma; the time for this stage depends on the depth of the DMD. Afterward, endothelium has to migrate to the gap between the broken DM edges and synthesize a new DM; the time required for this depends on the scale of the DM break. Injection of $\mathrm{C}_{3} \mathrm{~F}_{8}$ can hasten DM reattachment to the posterior stroma in the first step but not the second. ${ }^{23}$ In contrast, compression sutures can hasten both the first and second steps, probably by bringing the DM and stroma together and holding the edges of the tear in close apposition, thus enabling endothelial cells to rapidly seal and cover the lesion.

Acute corneal hydrops complicated by intrastromal cleft formation is considered a risk factor for delayed resolution, persistent edema, corneal perforation, and the development of stromal neovascularization. ${ }^{24}$ Corneal stromal neovascularization may decrease the long-term survival of a penetrating graft because of increased risk of rejection. Hydrops with clefts generally requires multiple intracameral gas applications. The clefts are usually connected to the anterior chamber by small gaps through which intracameral gas can easily enter into the corneal clefts. For this reason, compressive sutures with or without gas injection may be a better approach to treat acute corneal hydrops complicated by clefts. ${ }^{25}$
Eyes with wide DMD require more time for resolution of corneal edema, even with descemetopexy. ${ }^{23}$ Whereas localized and narrow separation of DM from the stroma following phacoemulsification typically resolves spontaneously, wide DMDs demand early recognition and timely intervention to achieve the best visual outcomes. If the DMD is wide, folded, or curled, or persists for a long time as in our third and fourth cases, surgical unfolding of the DM with full-thickness corneal sutures together with gas injection usually recommended.

In conclusion, we aimed to highlight the importance of the current optimal intervention methods in patients who suffer from DM ruptures or detachments. Although there are no established gold standards for the timing and nature of treatment, ophthalmologists should consider multiple factors when making management decisions, such as the location and duration of the detachment and tear, and the degree of anteroposterior separation from the posterior stroma. Descemetopexy with gas tamponade is an easy and effective treatment approach that can hasten the healing process and improve visual acuity. However, in complicated cases such as those with corneal clefts and chronic or large DMD, using compressive sutures with intracameral gas injection seems to be a better approach. Overall, surgical management of DM rupture/detachment can provide faster symptomatic relief and less healing-related corneal scarring with better visual rehabilitation and may alleviate the need for corneal transplant surgery in these patients. Further comparative studies with more patients are required to demonstrate the safety and efficacy of these procedures.

Informed Consent: Written informed consent was obtained from patients.

Peer-review: Externally peer reviewed.

\section{Authorship Contributions}

Surgical and Medical Practices: Ö.Ö.U., Concept: G.Ö., Ö.Ö.U., Design: G.Ö., Data Collection or Processing: G.Ö., Analysis or Interpretation: G.Ö., Ö.Ö.U., Literature Search: G.Ö., Writing: G.Ö., Ö.Ö.U.

Conflict of Interest: No conflict of interest was declared by the authors. 
Financial Disclosure: This work was partially supported by a research project from Ankara University.

\section{References}

1. Cibis GW, Tripathi RC. The differential diagnosis of Descemet's tears (Haab's striae) and posterior polymorpous dystrophy bands. A clinicopathologic study. Ophthalmology. 1982;89:614-620.

2. Rupture of Descemet's membrane secondary to presumed forceps trauma. Clin Eye Vis Care. 1995;7:195-201.

3. Guerriero S, La Tegola M, Monno R, Apruzzese M, Cantatore A. A case of Descemet's membrane rupture in a patient affected by Acanthamoeba Keratitis. Eye Contact Lens. 2009;35:338-340.

4. Guyer DR, Barraquer J, McDonnell PJ, Green WR. Terrien's marginal degeneration: clinicopathologic case reports. Graefes Arch Clin Exp Ophthalmol. 1987;225:19-27.

5. Ruiz RS, Saatci OA. Spontaneous Descemet's membrane tear and detachment. Arch Ophthalmol. 1991;109:20-21.

6. Tuft SJ, Gregory WM, Buckley RJ. Acute corneal hydrops in keratoconus. Ophthalmology. 1994;101:1738-1744.

7. Mackool RJ, Holtz SJ. Descemet's membrane detachment. Arch Ophthalmol. 1977;95:459-463.

8. Mulhern M, Barry P, Condon P. A case of Descemet's membrane detachment during phacoemulsificaton surgery. Br J Ophthalmol. 1996;80:185-186.

9. Rowson N, Dart J, Buckley R. Corneal neovascularisation in acute hydrops. Eye. 1992;6:404-406.

10. Shaw EL. Pathophysiology and treatment of corneal hydrops. Ophthalmic Surg. 1976;7:33-37.

11. Hirst LW, DeJuan E. Sodium hyaluronate and tissue adhesive in treating corneal perforations. Ophthalmology. 1982;89:1250-1253.

12. Macsai MS, Lemley HL, Schwartz T. Management of oculus fragilis in EhlersDanlos type VI. Cornea. 2000;19:104-107.

13. Hussin HM, Biswas S, Majid M, Haynes R, Tole D. A novel technique to treat traumatic corneal perforation in a case of presumed brittle cornea syndrome. Br J Ophthalmol. 2007;91:399.

14. Rajaraman R, Singh S, Raghavan A, Karkhanis A. Efficacy and safety of intracameralperfluoropropane (C3F8) tamponade and compression sutures for the management of acute corneal hydrops. Cornea. 2009;28:317-320.
15. Mohebbi M, Pilafkan H, Nabavi A, Mirghorbani M, Naderan M. Treatment of Acute Corneal Hydrops With Combined Intracameral Gas and Approximation Sutures in Patients With Corneal Ectasia. Cornea. 2020;39:258-262.

16. Zhao Z, Wu S, Ren W, Zheng Q, Ye C, Kim AD, Jhanji V, Wang MTM, Chen W. Compression sutures combined with intracameral air injection versus thermokeratoplasty for acute corneal hydrops: a prospective-randomised trial. Br J Ophthalmol. 2021;105:1645-1650.

17. Selver ÖB, Eğrilmez S. Diagnosis and Management of Descemet's Membrane Detachment: A Cause of Corneal Edema After Cataract Surgery. Turk J Ophthalmol. 2014;44:486-489.

18. Miyata K, Tsuji H, Tanabe T, Mimura Y, Amano S, Oshika T. Intracameral air injection for acute hydrops in keratoconus. Am J Ophthalmol. 2002;133:750752.

19. Panda A, Aggarwal A, Madhavi P. Management of acute corneal hydrops secondary to keratoconus with intracameral injection of sulfur hexafluoride (SF6). Cornea. 2007;26:1067-1069.

20. Shah SG, Sridhar MS, Sangwan VS. Acute corneal hydrops treated by intracameral injection of perfluoropropane (C3F8) gas. Am J Ophthalmol. 2005:3427-3429.

21. Sonmez K, Yasin P. Surgical repair of scrolled descemet's membrane detachment with intracameral injection of $1.8 \%$ sodium hyaluronate. Int Ophthalmol. 2011;31:421-423.

22. Jain R, Murthy S, Basu S. Anatomic and visual outcomes of descemetopexy in post-cataract surgery descemet's membrane detachment. Ophthalmology. 2013;120:1366-1372.

23. Basu S, Vaddavalli PK, Vemuganti GK, Ali H, Murthy SI. Anterior segment optical coherencetomography features of acute corneal hydrops. Cornea. 2012;31:479-485.

24. Feder RS, Wilhelmus KR, Vold SD, O'Grady RB. Intrastromal clefts in keratoconus patients with hydrops. Am J Ophthalmol. 1998;126:9-16.

25. Vohra V, Shetty R, James E, Kundu G, D'Souza S. Evaluating the safety and efficacy of compression sutures with intracameral perfluoropropane $(\mathrm{C} 3 \mathrm{~F} 8)$ in the management of acute corneal hydrops. Int Ophthalmol. 2021;41:20272031. 